\title{
Trapping of pulse injected ions in a radio-frequency quadrupole trap
}

\author{
O Chun-Sing and H. A. Schuessler \\ Departement of Physics, Texas A and M University, College Station, Texas 77843, U.S.A.
}

(Reçu le 24 juillet 1981, révisé le 16 octobre 1981, accepté le 20 octobre 1981)

\begin{abstract}
Résumé. - Le stockage des ions injectés du dehors à l'intérieur d'une cage quadrupolaire HF est étudiée. Par ceci la tension de confinement à haute fréquence est mise en circuit phase synchronisée. On utilise en particulier la méthode d'espace de phase pour dériver les conditions optimales. En principe il est possible de piéger les ions indéfiniment. Une efficience haute de piégeage, proche de l'unité, est possible. Une sélectivité d'énergie et de masse peut être aussi réalisée.
\end{abstract}

\begin{abstract}
The possibility of trapping ions from an externally injected pulsed ion beam in a radio-frequency quadrupole ion trap, with phase-synchronized switching-on of the driving field, was investigated. Phase space methods were used to derive the optimum conditions for permanently trapping the injected ions. A high trapping efficiency, close to unity, is possible and energy-selectivity as well as mass-selectivity of the stored ions can be obtained.
\end{abstract}

1. Introduction. - Novel spectroscopic investigations of charged atomic systems can be realized if externally generated ions are caught in flight and subsequently localized in a finite observation volume. In particular on-line spectroscopy of radioactive ions [1], highly charged ions [2], and exotic ions produced by any means are of interest. To investigate this possibility, the ion confinement properties of a radiofrequency (r.f.) quadrupole ion trap are evaluated for pulsed ion beams injected from the outside.

The r.f. ion trap $[3,4]$ consists of a central ring electrode, which is a hyperboloid of revolution, and two end-cap electrodes, which are complementary hyperboloids. The symmetric axis of the ion trap is defined as the $z$-axis. When a potential difference $U$ is maintained between the ring electrode and the end-cap electrodes, the rotationally symmetric potential distribution within the ion trap is of the form

$$
V=\frac{U}{2 r_{0}^{2}}\left[2 z^{2}-\left(x^{2}+y^{2}\right)\right] .
$$

Here $r_{0}$ is the equatorial radius of the ion trap. The two electric field components in the axial and the radial directions are of different signs which means that at any given time focusing action takes place in one direction and defocusing in the other. In general, the driving voltage $U$ for a r.f. ion trap consists of a constant dc bias $U_{0}$ and an oscillating voltage of peak amplitude $V_{0}$ and angular frequency $\Omega$

$$
U=U_{0}+V_{0} \cos \Omega t .
$$

The defocusing action changes alternately with the focusing action at the frequency of the driving r.f. field. As the result of the rapidly oscillating voltage between the trap electrodes, an average focusing force is produced which is proportional to the distance from the center of the device. Thus the r.f. quadrupole ion trap generates a good approximation of a three-dimensional harmonic potential well in which low-energy charges particles are confined. The high resolution and sensitivity of spectroscopic measurements on stored ions have been demonstrated [5]. However, most of the works relied on ions created inside the ion trap by either photoionization or electron impact ionization. The number of elements measurable using the stored ion spectroscopy is therefore rather limited. Trapping the ions after their creation external to the ion trap will make possible new spectroscopic investigations.

2. Trapping of injected ions. - Ions created externally can be injected into the trap in two ways. Either the trap is on all the time or the trap is off during the injection and is turned on afterwards. The first possibility has been considered elsewhere [6]. In the other 
mode of operation the ion trap voltages are turned off during ion injection. The driving voltages, including the d.c. bias and the r.f. voltage at a particular phase $\xi_{0}$, are switched on with a suitably chosen time delay $T_{\mathrm{D}}$ after the injection of an ion pulse. In contrast to the continuous ion injection mode, in the pulse injection mode the ions are already inside the trap when they are first subjected to the quadrupole storage fields. As a result, indefinite confinement of the injected ions is possible. For an ion to be confined for an indefinite period inside the ion trap the ion motion must be stable and have maximum displacements from the center of the ion trap smaller than the corresponding dimensions of the trap. The first condition is satisfied by choosing the driving voltages for an ion of chargeto-mass ratio $e / m$, so that the parameters

$$
a_{z}=8 e U_{0} / m r_{0}^{2} \Omega^{2} \text { and } q_{z}=4 e V_{0} / m r_{0}^{2} \Omega^{2}
$$

of the Mathieu equation, which governs the motion of the ions, are within the region of stability. The second condition can be satisfied if the initial position of the ion is within a certain critical volume to be evaluated using the phase space methods $[7,8]$. The critical volume has in general the shape of a rectangular box defined by the three stable zones along the $x$-, $y$-, or $z$-axis. The values of $u_{1,2}$, i.e. the limits of the stable zone, in the $u$-direction are given, for an ion initial velocity $\dot{u}_{0}$, by

$$
u_{1,2}=-\left(\frac{\alpha_{u}}{\gamma_{u}}\right) \dot{u}_{0} \pm \frac{1}{\gamma_{u}}\left(\frac{\gamma_{u}}{\beta_{u, \max }} u_{\mathrm{d}}^{2}-\dot{u}_{0}^{2}\right)^{1 / 2} .
$$

Here $u_{\mathrm{d}}$ is the corresponding trap dimension which is not to be exceeded. The phase space ellipse parameters $\alpha_{w}, \beta_{u}$, and $\gamma_{u}$ depend on the operation point of the ion trap and the initial phase $\xi_{0}$ of the r.f. voltage. The quantity $\beta_{u, \text { max }}$ is the maximum value of the parameter $\beta_{u}$ over all possible values of the initial phase for that particular operation point. For an ion to be confined

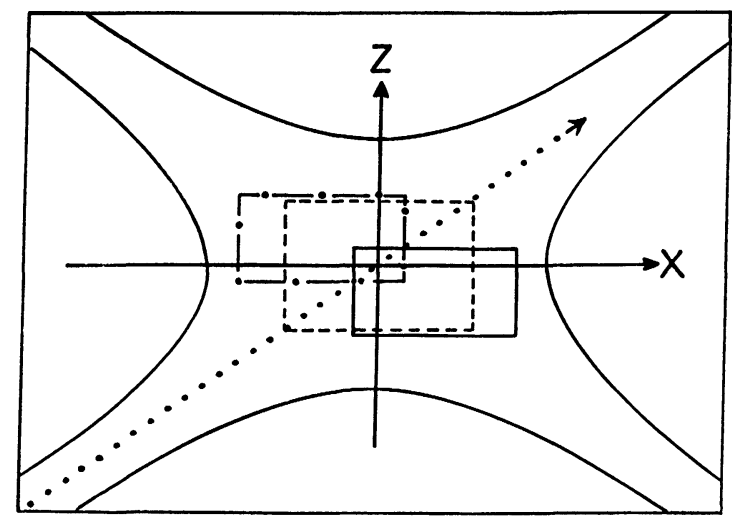

Fig. 1. - The cross-sectional areas in the $x z$ plane of the critical volumes for ion confinement in the case of gap injection at three different values of the initial r.f. driving-voltage phase $\xi_{0}$ : solid line, $\pi / 4$; dashed line, $\pi / 2$; dashed-dotted line, $3 \pi / 4$. The dotted line represents the unperturbed straight-line ion trajectory. The ion energy is $10 \mathrm{eV}$. in the rf trap the coordinates of the initial ion position must be within the critical volume bounded by the surfaces $x=x_{1,2}, y=y_{1,2}$ and $z=z_{1,2}$. For ions created inside the trap, this condition is not difficult to satisfy. But for injected ions the trapping quadrupole fields should be turned on at the right moment. Figure 1 depicts the cross-sectional areas of the critical volumes in the $x z$ plane at three different values of the initial r.f. voltage phase for injection of singly charged ions of mass number 100 and energy of $10 \mathrm{eV}$ into an ion trap, with operation parameters shown in table I, through the gap between the ring and one of the end cap electrodes. The two values of the time delay ' $\left(T_{\mathrm{D}}\right)_{1}$ and $\left(T_{\mathrm{D}}\right)_{2}$ at which the unperturbed straight-line ion trajectory intersects the surface of the critical volume determine the range of the time interval after which the quadrupole field, with the r.f. voltage at phase $\xi_{0}$, should be turned on for ion confinement. The time delay, $T_{\mathrm{D}}$, is measured from the instant when the leading ions of the pulse just arrive at the injection aperture. For an ion source at a certain distance $d$ from the injection aperture in addition a drifting time $T_{\mathrm{d}}$ should be included. Typical time delay intervals for ion confinement are several times larger than the oscillation period $2 \pi / \Omega, 1 \mu \mathrm{s}$ in the present case, of the driving r.f. field. The most favorable initial phase is the value of $\xi_{0}$ at which $\Delta T_{\mathrm{D}}=\left(T_{\mathrm{D}}\right)_{2}-\left(T_{\mathrm{D}}\right)_{1}$ is maximal. The optimum value of $\xi_{0}$ depends on the position of the injection aperture and the incidence direction. For ring electrode or end cap injection of the ion beam the injection aperture is supposed to be at the center of the corresponding electrode. The incidence direction is defined by $\theta$ and $\varphi$ where $\theta$ is the angle measured on a plane including both the incident beam and the electrode axis, and $\varphi$ is the aximuthal

Table I. - Operation parameters of the quadrupole rf trap.

Radial dimension, $r_{0}(\mathrm{~cm})$

0.54

Axial dimension, $z_{0}(\mathrm{~cm})$

0.38

Driving frequency, $\Omega / 2 \pi$ (MHz)

Ion mass, $m$ (amu)

Operation points

Stability parameters,

$q_{z}$

$a_{z}$

$q_{x}=q_{y}$

$a_{x}=a_{y}$

dc bias, $U_{0}(\mathrm{~V})$

ac trapping amplitude, $V_{0}$ (V)

Axial oscillation frequency, $\omega_{z} / 2 \pi=(\mathrm{kHz})$

Radial oscillation frequency, $\omega_{x} / 2 \pi=\omega_{y} / 2 \pi(\mathbf{k H z})$

Axial potential well depth, $e \bar{D}_{z}(\mathrm{eV})$

Radial potential well depth $e \bar{D}_{x}=e \bar{D}_{y}(\mathrm{eV})$

$$
1
$$

100

$$
\text { A }
$$

B

0.5

1.24

$-0.0313$

$-0.658$

$-0.25$

0.0156

$-0.62$

4.7

0.329

99

150

372

153

80

108

473

16.8

4.6 
angle measured from a reference axis on a plane perpendicular to the symmetry axis. On the other hand, for injection through the gap between the ring and one of the end-cap electrodes the injection aperture is at $\left(-2.04 r_{0}, 0,-1.43 r_{0}\right)$ which is the edge of the physical dimension of the quadrupole trap under consideration. The ion beam is along the asymptotic line of the hyperbolic cross-sections of the electrode surfaces. For a given operation point of the quadrupole trap the phase space ellipse parameters are calculated for all values of the initial phase $\xi_{0}$. In the present case, the trapping voltage $U$ is applied to the end cap electrodes. All values of the $\xi_{0}$ referred should be replaced by $\xi_{0}^{\prime}=\pi-\xi_{0}$ in the case when $U$ is applied to the ring electrode. The boundaries of the critical volume for ion confinement are then obtained for the given ion energy and injection direction. The time delay values $\left(T_{\mathrm{D}}\right)_{1}$ and $\left(T_{\mathrm{D}}\right)_{2}$ are calculated from the ion velocity and the coordinates of the intersection points of the critical volume and the unperturbed straight-line ion trajectory. For both the end cap injection and the ring injection cases the ion beam incidence angle $\theta$ is varied from $0^{\circ}$ to $80^{\circ}$ in steps of $5^{\circ}$. The azimuthal angle $\varphi$ is changed only from $0^{\circ}$ to $90^{\circ}$ in steps of $15^{\circ}$ in the ring injection case. Values of $\varphi$ in the other three gradiants can be obtained from symmetry considerations. On the other hand, in the end cap injection case the value of $\varphi$ is not important because of the cylindrical symmetry of the quadrupole configuration. For an ion beam of finite cross-section, ions at different positions on the beam cross-section will have different time delays to intersect the critical volume at slightly different positions. The crosssectional efficiency of ion confinement $\varepsilon_{\mathrm{ic}}\left(\tau, T_{\mathrm{D}}\right)$ is defined, for time delay $T_{\mathrm{D}}$ and ion pulse duration $\tau$, as the weighted sum of the percentage overlaps of the ion pulse with the respective time delay intervals for ion confinement. Trapping of the injected ions can be quite effective with $\varepsilon_{i c}$ close to unity for a well-collimated ion beam with a homogeneous population

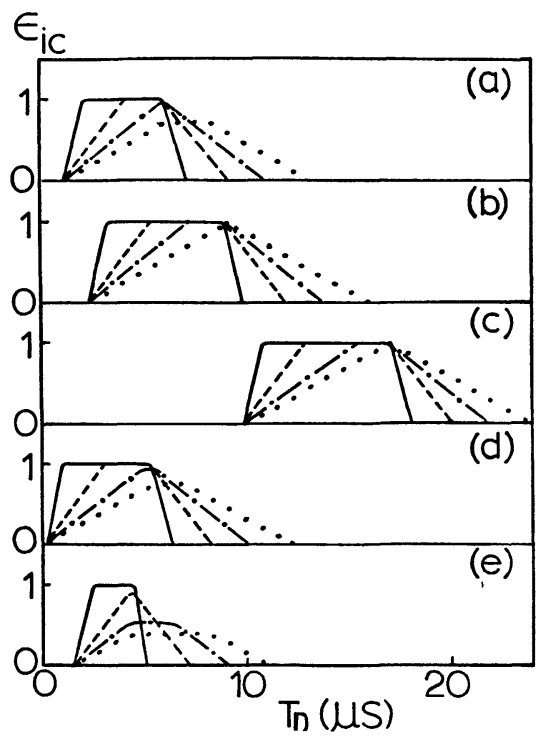

Fig. 2. - Cross-sectional efficiency $\varepsilon_{\text {ic }}$ of ion confinement for different ways of pulse injection of $10 \mathrm{eV}$ ions : (a) radial injection, (b) ring injection at $\theta=30^{\circ}$ and $\varphi=45^{\circ}$, (c) gap injection, $(d)$ end cap injection at $\theta=45^{\circ}$, and $(e)$ axial injection. The ion pulse durations are $1,3,5$, and $7 \mu$ s for the solid, dashed, dashed-dotted, and dotted lines, respectively.

distribution over a circular cross-section of $1 \mathrm{~mm}$ in diameter. For ions of mass number 100 with an energy of $10 \mathrm{eV}$ the most favorable beam incidence direction is $\theta=30^{\circ}$ and $\varphi=45^{\circ}$ for the ring injection case and $\theta=45^{\circ}$ for the end cap injection case. The ion confinement efficiency versus time delay curves at their respective optimum initial r.f. voltage phases for these cases as well as those for the radial, axial, and gap injections are shown in figure 2 . The corresponding peak ion confinement efficiencies are tabulated in table II for four different values of the ion pulse duration. The last two columns of table II depict the time

Table II. - The peak cross-sectional efficiency of ion confinement for an ion beam of an energy of $10 \mathrm{eV}$ and a circular cross-section of $1 \mathrm{~mm}$ diameter. The last two columns depict the corresponding time delay interval for ion confinement and the ion transit time, respectively.

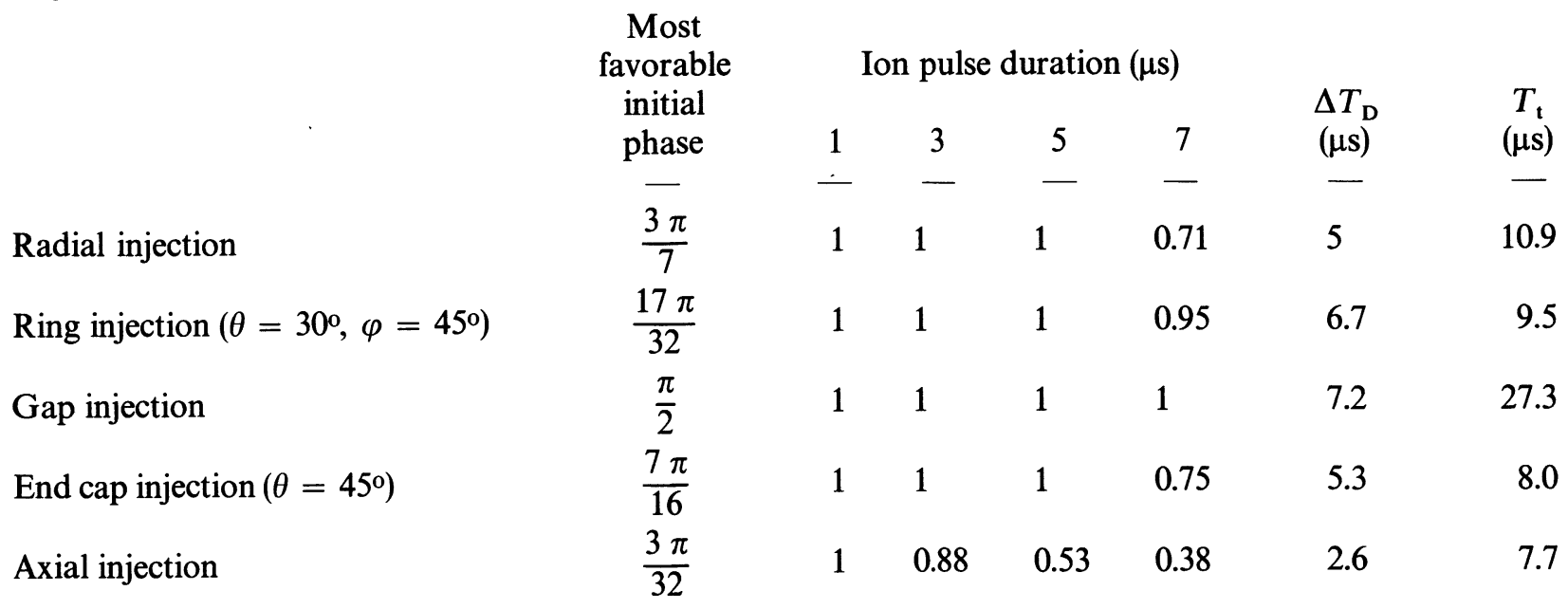




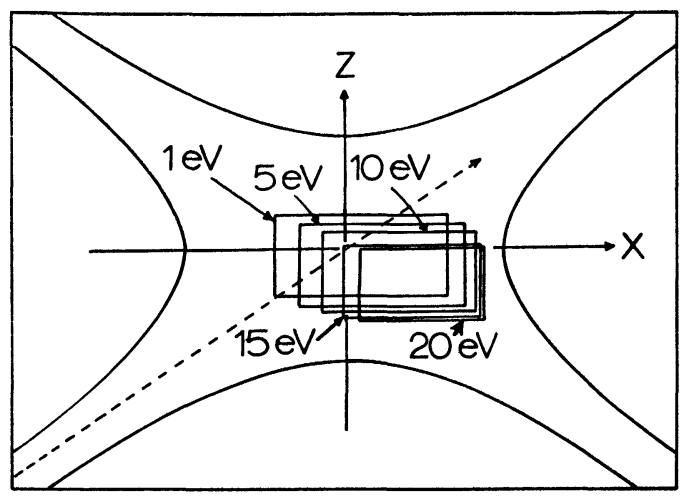

Fig. 3. - Cross-sectional areas in the $x z$ plane of the critical volumes for ion confinement in the case of gap injection at $\xi_{0}=\pi / 4$ for five different ion energies. The dashed line depicts the unperturbed straight-line ion trajectory.

delay interval for ion confinement, $\Delta T_{\mathrm{D}}$, and the ion transit time, $T_{\mathfrak{v}}$, respectively. Here the ion transit time is defined as the time interval the injected ion, at the beam center, takes to move from the injection aperture to a position where it hits the trap electrode or leaves the trap.

The limits of the stable zone, in the $u$-direction, $u_{1,2}$, depend on the corresponding ion initial velocity $\dot{u}_{0}$ in that direction. The stable zone in each direction is located at different positions and is of different size, which in turn leads to the dependence of the critical volume for ion confinement on the ion energy and the incidence direction. Figure 3 shows the relatively shifted cross-sections of the critical volumes for ions of different energies in the gap injection case. These cross-sections are of different sizes. Because of the difference in the ion velocities, the variation of the time delay interval for ion confinement is even larger, as depicted in figure 4. For an injected beam with a large energy spread complete ion trapping is not possible. Either the slower ions are not trapped when the quadrupole fields are turned on too early or the faster ions have already passed through their corres-

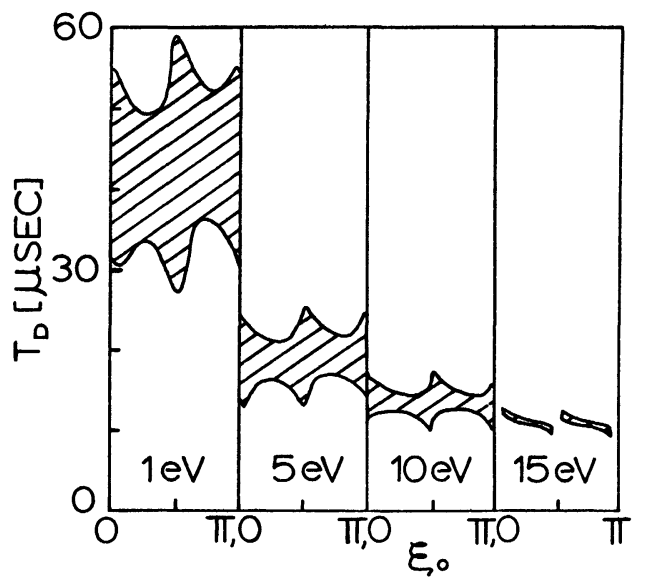

Fig. 4. - Time delay interval for ion confinement versus initial phase diagrams for gap injection of pulsed ion beams of different energies.
Table III. - The maximum peak overall cross-sectional efficiency of ion confinement for an injected ion beam of uniform energy distribution over the range of 0 to $20 \mathrm{eV}$.

Ion pulse duration $(\mu \mathrm{s})$

Radial injection

$\begin{array}{cccc}\frac{1}{-} & \frac{3}{-} & \frac{5}{-} & \overline{7} \\ 0.86 & 0.77 & 0.69 & 0.61 \\ 0.90 & 0.86 & 0.82 & 0.74\end{array}$

Ring injection

$$
\left(\theta=30^{\circ}, \varphi=45^{\circ}\right)
$$

Gap injection

$\begin{array}{llll}0.42 & 0.41 & 0.40 & 0.39\end{array}$

End cap injection $\left(\theta=45^{\circ}\right)$

$\begin{array}{llll}0.86 & 0.78 & 0.69 & 0.62\end{array}$

Axial injection

$\begin{array}{llll}0.68 & 0.54 & 0.44 & 0.38\end{array}$

ponding critical volumes for confinement when the quadrupole fields are turned on too late. In any case the overall ion trapping efficiency cannot reach unity. However, if the time delay value and the r.f. initial phase are chosen suitably, most of the incoming ions of different energies can be trapped simultaneously. Table III lists the maximum peak overall crosssectional efficiencies of ion confinement, for an injected ion beam with a uniform energy distribution over the range $0-20 \mathrm{eV}$, in some typical ion injection cases for different values of the ion pulse duration. For shorter ion durations reasonably high values of the overall ion confinement efficiency are possible.

3. Energy-selective ion storage. - The velocitydependence of the ion confinement property leads to energy selective storage of pulse injected ions. The magnitude and the width of the accepted ion energy can be selected. Energy-selective storage of ions can be achieved in two different ways. The first way is to choose suitable trap operation parameters to achieve a very narrow time delay interval for ion confinement at the ion energy of interest. The second way is to allow a field-free drift distance before ion injection and to take advantage of the spread in the times of flight for ions of different energies. In both ways an energy window of less than $1 \mathrm{eV}$ at an ion energy of $10 \mathrm{eV}$ can be obtained. Examples of the first way are tabulated in table IV. The second way is basically a sort of

Table IV. - Energy-selective storage of $10 \mathrm{eV}$ energy ions through suitable operation conditions.

$\begin{array}{lcc}\text { Injection aperture } & \begin{array}{c}\text { Midpoint of the } \\ \text { ring electrode }\end{array} & \begin{array}{c}\text { Apex of one } \\ \text { of the end } \\ \text { cap electrodes }\end{array} \\ \text { Incidence direction } & \begin{array}{c}\theta=15^{\circ} \\ \varphi=60^{\circ}\end{array} & \theta=70^{\circ} \\ \begin{array}{l}0.33 \pi \\ \text { Initial rf phase }\end{array} & \begin{array}{l}0.15 \pi \\ \text { Time delay }\end{array} & 5.486 \mu \mathrm{s} \\ \begin{array}{l}\text { Peak confinement } \\ \text { efficiency }\end{array} & 68 \% & 100 \% \\ \begin{array}{l}\text { Energy width at half } \\ \text { maximum effi- } \\ \text { ciency }\end{array} & 0.3 \mathrm{eV} & 0.28 \mathrm{eV}\end{array}$


time-of-flight device. The energy resolution depends on the allowed field-free drift distance and the initial spatial spread of the ion creation region. In addition, the ultimate energy resolution is limited by the time delay interval for ion confinement which defines the best time resolution obtainable. In the gap injection case with $\xi_{0}=0.3 \pi$, a drift distance just over $5 \mathrm{~cm}$ is needed to achieve an energy resolution of 17 at an ion energy of $10 \mathrm{eV}$, i.e. an energy window $\Delta E=0.6 \mathrm{eV}$. For the purpose of energy-selective ion storage the value of the r.f. voltage initial phase should be such that the corresponding time delay interval for ion confinement is minimal at the ion energy of interest. However, once the ions are confined in the r.f. quadrupole trap, their energy is changed to a value which is typically a small fraction of the effective trapping potential well depth and is independent of their energy before confinement.

4. Mass-selective ion storage. - Another result of the velocity dependence of the ion confinement properties of the r.f. quadrupole trap is the possibility to selectively store ions of a given isotope from a beam of a given energy but consisting of ions of different isotopes of the same element. The evaluation of the mass-selective storage properties of the r.f. quadrupole trap for injected ions is more complicated than the corresponding case of the energy-selective storage because the ion trap operation point is mass dependent. An example is shown in figure 5. The result of the massselective storage calculation is depicted in figure 6 where $d$ is the allowed field-free drift distance before ion injection, and $\Delta M$ is defined as the total width of the ion confinement efficiency curve at $10 \%$ of the peak efficiency. The thin horizontal lines in figure 6 represent the required values of the mass width and the mass resolution at an ion mass of 100 to separate ions of isotopes of masses 99 and 101. Similar to energyselective ion storage, for high mass resolution the initial phase of the r.f. voltage must be chosen such that the time delay interval for ion confinement is minimal at

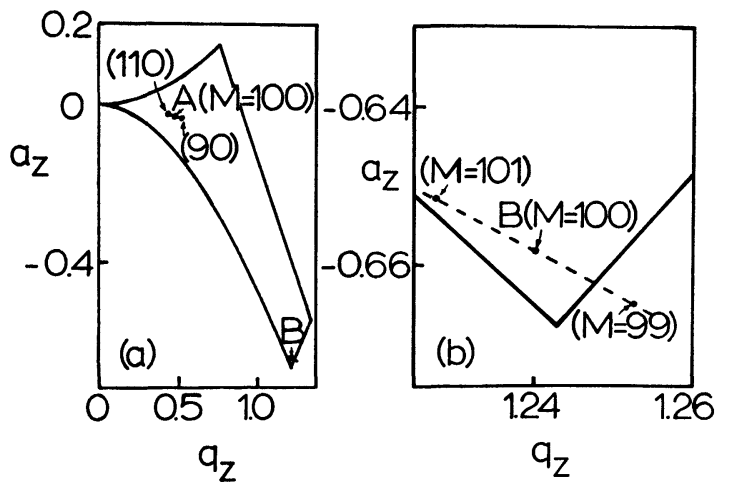

Fig. 5. - (a) Stability diagrams for a r.f. quadrupole trap showing the positions of the operation point for ions of different masses. (b) Magnified stability diagram near the mass-selective tip. For ions of mass 100 the operation points $\mathrm{A}$ and $\mathrm{B}$ are $\left(a_{z}, q_{z}\right)=(-0.0313,0.5)$ and $(-0.658,1.24)$, respectively.
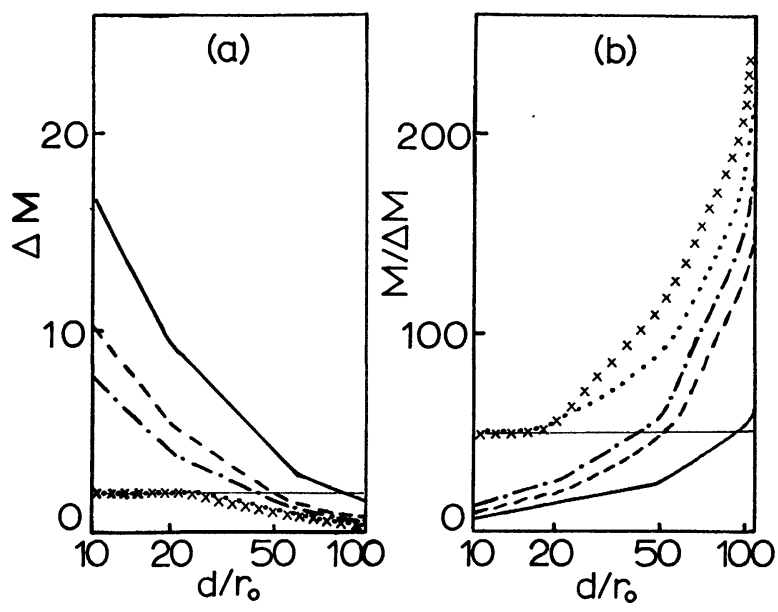

Fig. 6. - (a) The mass width and (b) the mass resolution at an ion mass of $100 \mathrm{u}$ are plotted against the drift distance between the ion creation region and the trap injection aperture. The solid, dashed, and dashed-dotted lines are for radial, axial, and gap injection, respectively, at the trap operation point $\left(a_{z}, q_{z}\right)=(-0.313,0.5)$. The dotted and the crossed lines are for radial and axial injection at the operation point $(-0.658,1.24)$.

the ion mass of interest. In this way mass-selective storage is no longer limited to only the mass-selective tip of the stability region but instead is possible for almost any allowed operation point of the ion trap.

5. Effects of different operation conditions. Because of the trap operation point dependence of the phase-space ellipse parameters, an example is depicted in figure 7, the critical volumes are expected to be quite different when the trap operation point is changed. This in turn introduces large variations in the ion confinement properties. In general, operating the ion trap at an operation point close to the boundaries of the stability region leads to smaller values for the time delay intervals for ion confinement. Higher energy-selectivity and mass-selectivity are obtained but at an expense of a lower ion confinement efficiency.

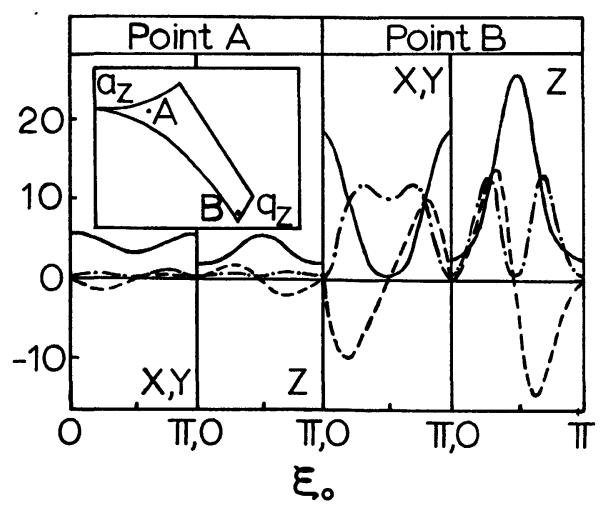

Fig. 7. - The phase space ellipse parameters as functions of the initial phase for two operation points of the quadrupole trap. The values of $\left(a_{z}, q_{z}\right)$ are $(-0.313,0.5)$ for point $\mathrm{A}$ and $(-0.658,1.24)$ for point $B$. The dashed, solid, and dashed-dotted lines depict the parameters $\alpha, \beta$, and $\gamma$, respectively. 
The results presented here can be used for other traps, of different size characterized by $r_{0}^{\prime}$ and operated at a different angular frequency $\Omega^{\prime}$, for an ion of different mass $m^{\prime}$ and charge $e^{\prime}$ after some scaling procedures. The d.c. bias $U_{0}$ and the r.f. driving amplitude $V_{0}$ should be changed to arrive at the same values for the stability parameters to ensure the same operation point of the quadrupole trap. Both of these voltages are modified by a factor $\left(m^{\prime} r_{0}^{\prime 2} \Omega^{\prime 2} / e^{\prime}\right) /\left(m r_{0}^{2} \Omega^{2} / e\right)$. In addition, the ion energy must be scaled according to $\left(m^{\prime} r_{0}^{\prime 2} \Omega^{\prime 2}\right) /\left(m r_{0}^{2} \Omega^{2}\right)$ to obtain the same ion injection velocity used in this report. In general, for ions of the same mass and charge, a trap of a larger size, but operated at the same r.f. frequency and the same operation point has equivalent ion confinement properties for much higher ion energy. However, the required driving quadrupole fields should be of correspondingly increased amplitudes.

Acknowledgments. - This work is supported by the U.S. National Science Foundation under Grant Number Phy. 7909099, by the U.S. Department of Energy under Contract Number DE-AS05-80 ER 19578, and by the Center for Energy and Mineral Resources at Texas A and M university.

\section{References}

[1] Schuessler, H. A., Phys. Today 34 (1981) 48.

[2] Gray, T. J., Cocke, C. L., Justiano, E., Phys. Rev. A $22(1980) 849$.

[3] Dehmelt, H. G., Adv. At. Mol. Phys. 3 (1967) 53; 5 (1969) 109.

[4] Schuessler, H. A., in Progress in Atomic Spectroscopy, Hanle, W. and Kleinpoppen, H., eds (Plenum) 1978, pp. 999.
[5] Schuessler, H. A., Fortson, E. N., Dehmelt, H. G., Phys. Rev. 187 (1969) 5.

[6] O, C. S., Schuessler, H. A., J. Appl. Phys. 52 (1981) 1157.

[7] Baril, M., Septier, A., Revue Phys. Appl. 9 (1974) 505.

[8] Dawson, P. H., Lambert, C., Int. J. Mass Spectrom. Ion Phys. 16 (1975) 269. 